\title{
Vehicle Maintenance Notification System Using RFID Technology
}

\author{
Fathollah Amouzegar and Ahmed Patel
}

\begin{abstract}
Effective utilization of Radio Frequency Identification (RFID) technology between vehicle and base station over a wireless channel to facilitate vehicle maintenance monitoring and vehicle authentication is proposed. This system is able to automatically register the vehicles in highways or gas stations and inform the user about the vehicle maintenance states. The details of the required services are sent to the user at the clocking of the mileage of the vehicle approaching time for service or already due to be serviced. The implementation is divided into two parts; the vehicle module and the base module. The proposed system attempts to solve the communication coverage problem. It also increases the supported range to solve the home use limitation and improve the accuracy of the data transferred by using Electronic Control Unit (ECU).
\end{abstract}

Index Terms-RFID, communication, vehicle maintenance, tag, Wi-Fi.

\section{INTRODUCTION}

The application of vehicle service and maintenance has a significant role in informing the user that the vehicle is due for service at regular intervals to ensure prime operating condition of the car. Most vehicle users are careless in paying enough attention to the maintenance schedule and obeying servicing due date. The on-time maintenance system can decrease the service time and prevent scar malfunction of the engine other parts. This is why it is important for car owners and users to pay attention to the service maintenance schedule in a timely manner. At a corporate level, insufficient attention to the proper servicing has direct influence on the financial factors of vehicle fleet and logistical operation of their business. Otherwise, it may lead to both unnecessary repair cost and loss of revenue respectively.

The problem is that there is no effective indicator or warning maintenance system in most vehicles in terms of a signal to remind drivers that the mileage of their vehicles is approaching time for service.

\section{LITERATURE REVIEW}

Recently, some technologies have been changed from

Manuscript received October 23, 2012; revised November 17, 2012.

Fathollah Amouzegar is with the School of Computer Science, Centre of Software Technology and Management (SOFTAM), Faculty of Information Science and Technology (FTSM), Universiti Kebangsaan Malaysia (UKM) (e-mail: fateh_amouzegar@yahoo.com).

Ahmed Patel is with the School of Computing and Information Systems, Faculty of Science, Engineering and Computing, Kingston University, Kingston upon Thames KT1 2EE, United (e-mail: whinchat2010@sgmail.com). traditional calendar based maintenance to e-maintenance via interaction through the use of data communication technology, such as the Electronic Product Code (EPC) and RFID [1]. For example, Hiraoka et al. display the method for a sector agent that made a recommendation on part maintenance based on the gathered data from historical data and internet [2]. In addition, Djurdjanovic and Lee used multi sensor machine estimation and forecast to realize predictive vehicle maintenance condition by reducing assessment of reading multiple sensors to examine significant properties of the process or machinery in a networked and tether-free environment [3].

Moreover Preethichandra (2000) made multifunctional sensor for measuring viscosity, temperature, capacitance and cleanness of engine oil to make an evident choice on their maintenance state [4]. Mazlan et al. (2008) specified the in-car maintenance system based on receiving satellite signals to compare the mileage data with database for forecasting the service time. This system used GPS toolkit software to measure the speed of the vehicle in $\mathrm{Km} / \mathrm{h}$ format to take out the distance travel information and inform the user for next service time [5]. Kirchgessner et al. proposed the Wireless ECU Monitoring System (WEMS) used to monitor and manage engine features as engine RPM, oxygen sensor and air flow rate in order to optimize engine functionality. WEMS is able to provide access to ECU data and engine condition to track the maintenance states via a home PC, that is limitation of supported range for this system [6]. Boss et al. invented system that used passive RFID device for tracking vehicle maintenance. The RFID device is embedded within the vehicle's computer system in combination with sensors to track other components and parts for servicing wirelessly [7].

Park and Cho used RFID system to measure oil performance that is dependent on imperfect burning engine, pressure and high temperature. This system includes a capacitive IDT (Inter Digit Type) sensor, passive RFID tag and RFID reader using a low frequency of $125 \mathrm{KHZ}$ [8]. Furthermore, there are many publications related to engine oil maintenance and prediction of the maintenance problems, but hardly any forecasting the vehicle maintenance service needs, which the research reported in this paper attempts to provide.

The requirements for proposed system are:

1) To improve the data transmission accuracy by using serial communication between ECU and RF Modem.

2) To solve home use limitation and significantly increases the supported range.

3) Improve the frequency performance.

4) To provide internal service maintenance information to the user within the vehicle surrounds.

5) To provide the vehicle maintenance module to 
communicate with the base RFID station system to alert vehicle user of service maintenance schedule via SMS onto their mobile handset.

6) To decrease the implementation cost.

The architecture of the proposed system is based ECU monitoring system used active RFID technology for monitoring the vehicle maintenance states. In addition the proposed system used ECU data for vehicle maintenance instead of RFID tag sensor that are expensive when embedded in engines and serviceable parts.

\section{EVAlUAtion of USABLE TeChNOLOGIES}

There are six technologies that are usable for proposed system, so it is necessary to evaluate these technologies to choose the suitable one to achieve the research objective. These technologies are explained and the main three of these technologies named as Wi-Fi RFID, Ultra Wide Band (UWB) RFID and Active RFID are compared in details.

\section{A. Wi-Fi RFID (2.45 GHz)}

Nowadays, the Wi-Fi networks are available everywhere, and tags with usability at IEEE 802.11x standard are provided. The benefit of this usability seems to be using the existing Wi-Fi infrastructure in implement systems based on Wi-Fi tag technology. The disadvantages are the tag lifespan decreased, because of increasing of the data overhead of using the $802.11 \mathrm{x}$ protocol the power consumption is increased, vendors do not follow a uniform usage at the $802.11 \mathrm{x}$ standard. The comparison of Active RFID and Wi-Fi RFID is tabulated in Table 1.

TABLE I: ANALYZE THE IMPORTANT FACTORS FOR ACTIVE RFID

\begin{tabular}{lll}
\multicolumn{2}{c}{ TECHNOLOGY AND WI-FI RFID } \\
\hline \hline Factors & Active RFID & Wi-Fi RFID \\
Cheaper Tag Price & Yea & No \\
Smaller Tag Size & Yes & No \\
Longer Read Range & Yes & No \\
Higher Tag Density & Yes & No \\
Work better around metal & Yes (433 MHz & No (2.4 GHz High \\
and liquid & low frequency) & frequency) \\
Granularity Cost & Cheap & Very Expensive \\
& & (infrastructure \\
& & required) \\
Battery Life & Long & Short \\
Secure tag- Sealed \& & Yes & No \\
robust & & \\
Infrastructure Costs & Low & High \\
Maintenance Cost & Low & High \\
Frequency adaptability & 433MHz: & 2.4 GHz: obstacles \\
& friendlier with & are numerous, \\
& obstacles/ no LOS & accurate reading \\
Software to use & & requires LOS \\
Network Traffic & Open (Unlimited) & Forced to buy by \\
& & vendor \\
\hline
\end{tabular}

\section{B. ZIGBee (IEEE 802.15.4), Bluetooth and GPS}

The arena of active RFID have other technology options such as Zigbee (IEEE 802.15.4), Bluetooth and GPS.

1) ZIGBEE (IEEE 802.15.4)

The Zigbee can be used in the active RFID areas. Because it is useful for data communications at bi-directional via mesh network with low power. It offers the range up to 70 meters, and it is usable in traditional active RFID areas. However,
Zigbee is not an ideal technology for identifying location and it is not yet widely available. Besides, the Zigbee standards for RFID are not still completely developed.

\section{2) Bluetooth}

The Bluetooth can also be used in active RFID arena. It has short-range wireless networking standard that it is commonly found in PC accessories and mobile phones, and it is also looking for offering a platform for asset tracking applications. Its shorter range, consumes more power and the data transmission rate is slower than Wi-Fi. It is proper for niche application that the battery life span is not a critical factor.

\section{3) $G P S$}

GPS is another technology in active RFID arena; however, vehicle fleet and transport container tracking uses GPS already widely. This technology has high power consumption, it is relatively expensive, and it has some limitations in working indoors. These limitations make it inappropriate for the most of asset tracking and monitoring applications.

\section{Ultra Wide Band (UWB) RFID}

Since 2003, the UWB technology with release of the broad spectrum of microwave frequency by FCC is available for deployment with RTLS system. The UWB key advantage is precise locating of a tag within a three dimensional space. The infrastructure cost for UWB technology is a disadvantage that is very expensive as compared with all other active RFID technologies. UWB offers a maximum range of around 50 meters. Other comparison futures between Active RFID and UWB are presented in Table 2.

TABLE II: ANALYZE THE IMPORTANT FACTORS FOR ACTIVE RFID TECHNOLOGY AND UWB RFID

\begin{tabular}{lll}
\hline \multicolumn{1}{c}{ Factor } & \multicolumn{1}{c}{ Active RFID } & UWB RFID \\
Cheaper Tag Price & Yea & No \\
Smaller Tag Size & Yes & No \\
Longer Read Range & Yes & No \\
Higher Tag Density & Yes & No \\
Work better around metal & Yes (433 MHz low & No $(3.1 \mathrm{GHz}$ \\
and liquid & frequency) & High \\
& & frequency) \\
Granularity Cost & Cheap & Very \\
& & Expensive \\
Battery Life & Long & Short \\
Secure tag- Sealed \& robust & Yes & No \\
Infrastructure Costs & Low & High \\
Maintenance Cost & Low & High \\
Frequency adaptability & 433MHz: friendlier & $2.45 \mathrm{GHz} \& 6$ \\
& with obstacles/ no & $-8 \mathrm{GHz}$ \\
& LOS & \\
Software to use & Open (Unlimited) & Forced to buy \\
& & by vendor \\
Network Traffic & Low & \\
\hline \hline
\end{tabular}

According to the cost comparison of these main three techniques for the proposed system, the conventional active RFID offers the lowest cost in comparison among the others.

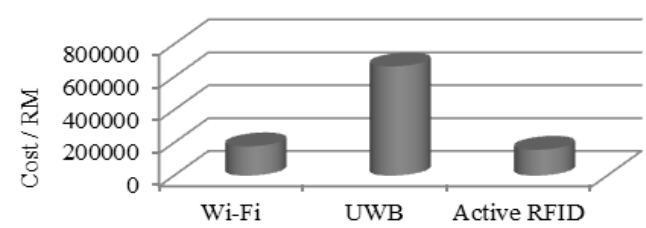

Fig. 1. Differences in costs for three main active RFID technologies

The UWB proposition as illustrated at Fig. 1, is the highest but when the accurate location of tag is paramount, UWB is 
the best suited to the application. So very seldom with conventional actives RFID system is considered together.

According to the above comparison, the suitable choice of available technologies for implementing the proposed system is an active RFID technology that is comprised and explained in details.

\section{Protocol Algorithm Behavior}

The Protocol algorithm behavior of the proposed system is displayed at Fig. 2.

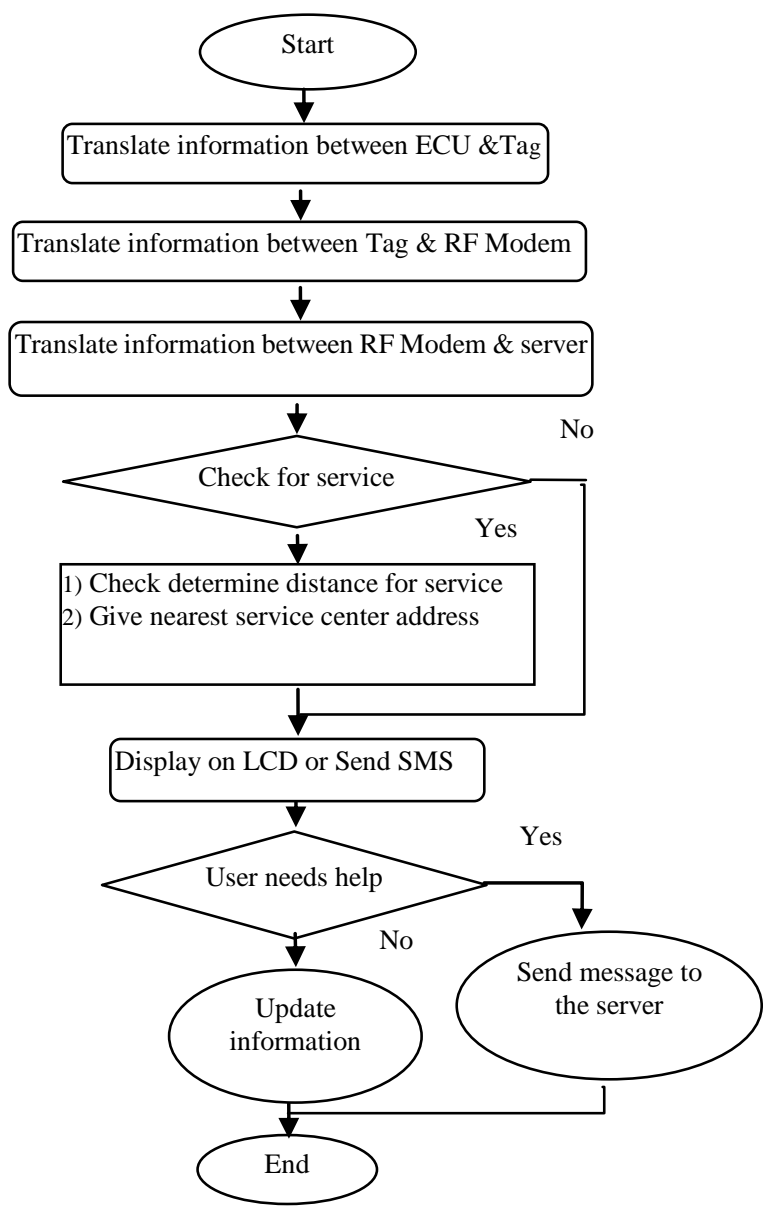

Fig. 2. Protocol behavior of the proposed system

This figure demonstrates the relation and workflow of these devices in a flowchart.

\section{Design AND Simulation OF PROPOSED System}

The automated vehicle maintenance monitoring system uses an active RFID tag [9] to exploit surrounding transmission frequency range within the car to inform the user by displaying the maintenance messages on the LCD inside the vehicle. Similarly, if appropriate tag readers are placed at facilities like road tolls can be utilized to interact with the base station system to send SMS messages to the car user. The proposed system design is illustrated in Fig. 3. The design is divided into two parts: (i) the vehicle module and (ii) the base station system module. Fig. 3 shows the communication process between the vehicle module and base station system module.

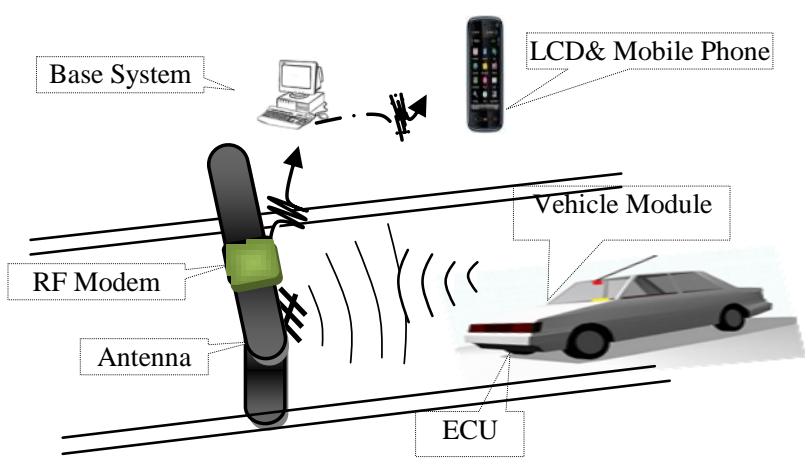

Fig. 3. Design of RFID vehicle maintenance system

\section{A. Base Module}

The base module can be installed at the gas stations or the barriers of Toll Plazas. The base module function is to register the vehicles in their range, to aware the users of the vehicle's condition and to give the alert messages if the vehicle achieves to maintenance service time. The schematic base module device is displayed at Fig. 4.

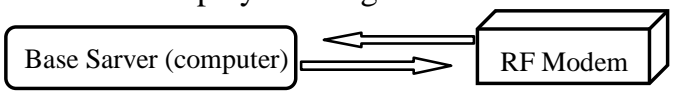

Fig. 4. Base module

The base module is programmed in order to send continuously an interrogation message over its range. If it receives any vehicle data, it measures the received data with the pre-stored data in its database. If the vehicle achieves to maintenance service time, the base sends a message to the vehicle module. Furthermore, the base station is able to send short messages in the chat area, which is usable for sending out broadcasts or other road related warnings. In addition, access to the vehicles related data is facilitated for the users, which these data are as registration numbers, owner's names, distance travelled and maintenance information. This system is designed to be simple and user friendly.

\section{B. Vehicle Module}

The vehicle module uses an active tag and RF Modem with $300 \mathrm{~m}$ of range that just by replacing the RF modem with another modem the range can be extended. The cost difference between the two modems is nominal. The RF modems require having frequency band ranging from 902 to $928 \mathrm{MHz}$, than the effect of RFID shielding is minimum at this frequency range. The required device in vehicle module is displayed at Fig. 5.

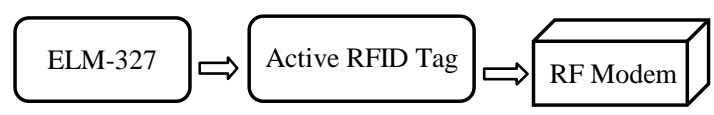

Fig. 5. Vehicle module

The designed active tag uses 8051 microcontroller [10]. This microcontroller is easily reprogrammable, which is the advantage for a future upgrade. It contains the data consists of registration number, chassis number, engine number or owner's name. Microcontroller operates at $+5 \mathrm{~V}$; whereas, RF modem communicates through serial port at $+9 \mathrm{~V}$. Therefore, there is a need to convert the signal levels going through RF modem to microcontroller and vice versa. MAX-232 IC is used to convert voltage levels from $+5 \mathrm{~V}$ and $+9 \mathrm{~V}$, it has been 
placed between microcontroller and RF modem. Data coming through RF modem is converted from $+9 \mathrm{~V}$ to $+5 \mathrm{~V}$. Similarly data coming through microcontroller is converted from $+5 \mathrm{~V}$ to $+9 \mathrm{~V}$. The RF modem communicates with base via a wireless channel, and ssit operates in ISM (Industrial, Scientific \& Medical) $900 \mathrm{MHz}$ band license-free 902-928 $\mathrm{MHz}$ frequency band manufactured under ISO 9001:2000 registered standards. The modulation scheme used is Frequency Hopping Spread Spectrum [11].

The ELM327 OBD scanner is used for communication between active tag and vehicle ECU. This device has two ways to communicate data which originate on vehicles on-board computer. These ways are RS-232 serial port, and USB port. The RS-232 is used to connect active tag to the ELM327 to send requests of data such as vehicle's speed, Air filter, Brake fluid, Fuel-line filter and another data from ECU to the active tag.

\section{RF Modems}

The implementation needs the reliable method of two-way communication over a wireless channel. Otherwise the active tag requires long range and low power consumption, that's why the RF modems with follows specification, chooses. The RF modem specifications [12] are: the indoor range is up to $90 \mathrm{~m}$ and outdoor range is up to $300 \mathrm{~m}$, receiver sensitivity is $-108 \mathrm{dBm}$ (9600 Baud), $-104 \mathrm{dBm}$ (38400 Baud). The transmitter power output is $4 \mathrm{~mW}-50 \mathrm{~mW}$ that is low power. The RF Modems are able to communicate with an option in frequency hopping spread spectrum (FHSS) mode or single frequency mode over the $900 \mathrm{MHz}$ frequency band. The modems are able to support successive data stream at 38400 bps [13]. Their specifications satisfy the communication requirements.

\section{Base Module Implementation}

The base module is implemented with connecting the RF modem by serial port to the computer [14]. Managing the data communication through the user interfacing is provided by Digi International. The base station which is displayed at Fig. 6 makes the operator to give the list of vehicles and displayed the data of vehicle registered with overview of area in range.

\section{E. Vehicle Module Implementation}

The vehicle module is installed in vehicle so at the time that the vehicle enters in the range of the base module, it receives the interrogation message. Then the stored data in microcontroller such as registration number, engine number or owner's name would be sent to the base. The received data from vehicle is checked by the base if the data is valid the vehicle registered. Then, it sends an authentication message to vehicle after registration. The vehicle module receives the authentication message and sends its specific notification message (vehicle's speed, Air filter, Brake fluid, Fuel-line filter data etc.) to the base module to check the maintenance service and to update the data. The base evaluates the states of vehicle maintenance and sends an informing message to the vehicle. This message is displayed to user on the LCD that interfaced with 8050 microcontroller used crystal frequency of $3.5 \mathrm{MHz}$ [15]. The vehicle module algorithm is illustrated at Fig. 7.

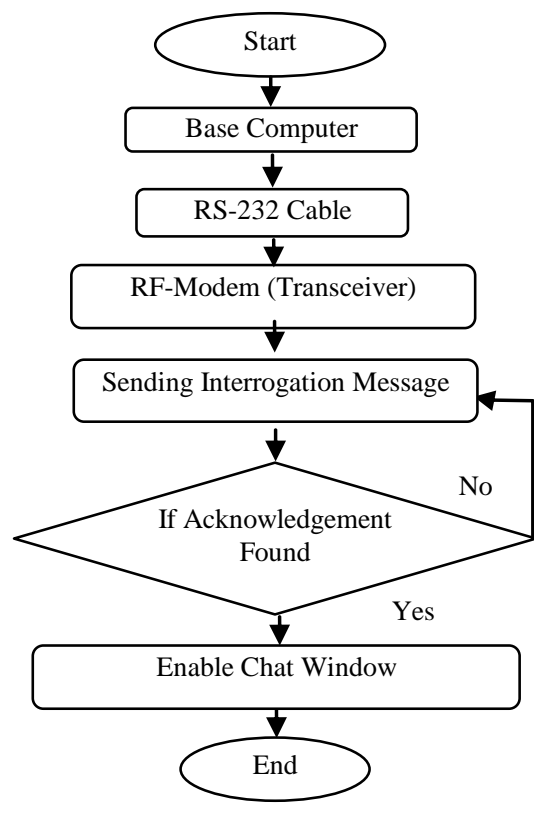

Fig. 6. Base station algorithms

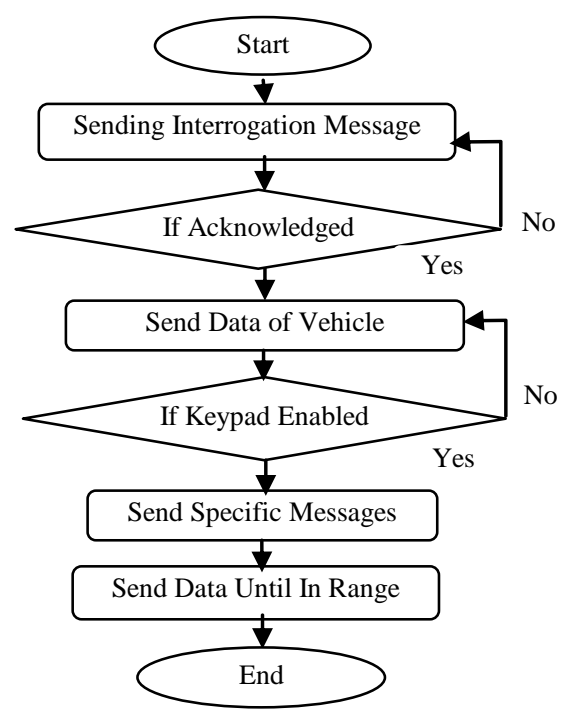

Fig. 7. Vehicle module algorithm

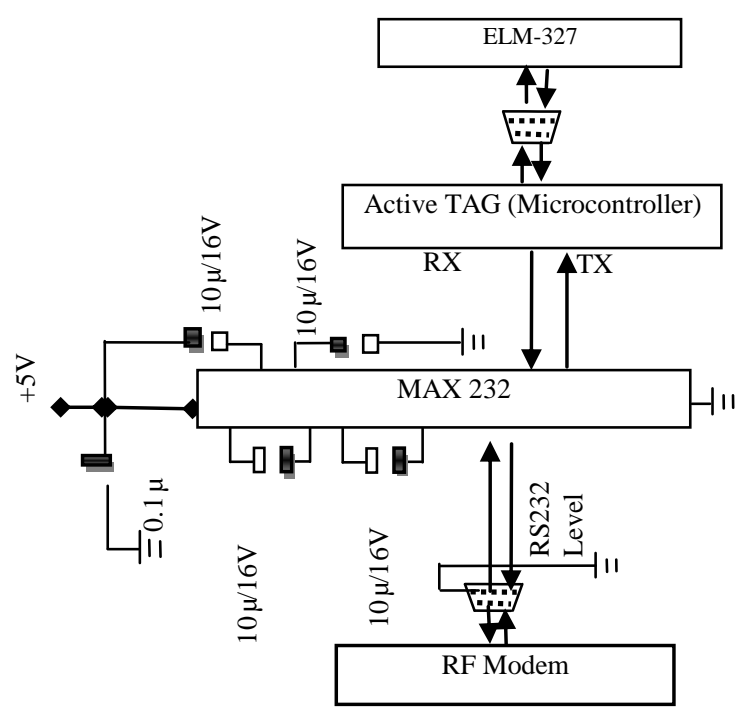

Fig. 8. Vehicle module circuit diagram 
The circuit diagram illustrated the communication between these devices, the data is transferred by RS232 serial port to the active tag, then active tag communicate with RF Modem by RS232 serial port. MAX232 is installed between active tag and RF Modem to convert the voltage between tag and RF Modem. The circuit diagram of vehicle module is displayed in Fig. 8.

This circuit diagram is used in the vehicle module to transmit the ECU maintenance data to the RF modem for communication between the vehicle and the off-site base module.

\section{COMPARATIVE ANALYSIS OF PROPOSED SYSTEM WITH COMPARABLE SYSTEM}

The comparison and evaluation of current vehicle maintenance systems with proposed system explained to demonstrate the research achievement.

\section{A. Supported Range}

The proposed system offered a longer range than vehicle maintenance systems that are currently employed. The current range is extended by proposed system of 90 meters to 300 meters, to solve the home use limitation. Furthermore, the offered range can be increased from 300 meters to 900 meters by using Digi International Xcite modems operating at 2.4 $\mathrm{GHz}$ band.

\section{B. Data Accuracy}

The proposed system improves the data accuracy by using the serial communication between ECU and RF modem to deal with the trouble arises by noise [8].

\section{Cost}

The proposed system implementation cost is less than other vehicle maintenance systems using RFID technology available in the market.

\section{Frequency}

The reasons to choose these frequencies [433 MHz, 868 $\mathrm{MHz}$ (Europe) and $915 \mathrm{MHz}$ (USA)] are stated as these are the most common freely and unlicensed UHF frequency, another reason is that they are easier to shield high frequency systems than lower frequency system.

\section{E. Memory}

The vehicle module is including the active tag that is equipped with microcontroller, and it is able to enhance the capabilities at interface with an external memory.

According to this comparison the proposed system improved the frequency performance and solved the communication coverage problem by using the serial port communication. In addition the supported range significantly increased to solve the home use limitation, and implementation cost is less than other RFID vehicle maintenance systems.

\section{CONCLUSION}

The hardware and software solution for vehicle maintenance service based on RFID is successfully proposed. This system used the ECU data to determine the vehicle maintenance state and improved the data accuracy. In addition used RF Modems to increase the frequency performance in operation, and significantly enhanced the supported range. Finally, the proposed system can accept a chat request in range, if vehicle needs help or any type of maintenance feedback. This system is able to use in other applications as automatically toll collection system, useful for combat theft detection, traffic patterns broadcast and other necessary indications to vehicles to ensure the smooth flow of traffic.

\section{REFERENCES}

[1] L. Lee, "Using wireless technology and the internet for predictive maintenance," Hydrocarbon processing, vol. 80, pp. 77-89, 2001.

[2] H. Hiraoka, N. Iwanami, Y. Fujii , T. Seya, and H. Ishizuka, "Network agents for life cycle support of mechanical parts," In Proc. of $3 \mathrm{rd}$ International Symposium on Environmentally Conscious Design and Inverse Manufacturing, pp. 61-64, 2003.

[3] D. Djurdjanovic, J. Lee, and J. Ni, "Watchdog agent-an infotronics-based prognostics approach for product performance degradation assessment and prediction," Advanced Engineering Informatics, vol. 17, 109-125, 2003.

[4] D. Preethichandra and K. Shida, "Actual condition monitoring of engine oil through an intelligent multi-functional sensing approach," in Industrial Electronics Society, vol. 4, pp. 2383-2387, 2000.

[5] E. M. Mazlan, F. Mohamad Nasir, L. Ab Rahim, R. Kasbon, S Mahamad, and J. Ahmad, "In-Car maintenance system: An Integrated vehicle information system," presented at the Information Technology and Multimedia, 2008.

[6] N. Houghton, Kirchgessner, and Robert, "A Wireless ECU Monitoring System Team WEMS," University of Florida, 2009.

[7] G. J. Boss, P. G. Finn, R. A. Hamilton, B. M. O'Connell, J. W. Seaman, and K. R. Walker, "Tracking vehicle maintenance using sensor detection," 2010.

[8] J. Cho and S. Park, "Capacitive sensor for automotive engine oil degradation using wireless network," presented at the International Symposium on Advanced Packaging Materials: Microtech, 2010.

[9] W. J. Yoon, S. H. Chung, and S. J. Lee, "Implementation and performance evaluation of an active RFID system for fast tag collection," Computer Communications, vol. 31, pp. 4107-4116, 2008.

[10] J. Stewart, “8051 Microcontroller: Hardware, software and interfacing $2 \mathrm{E}, " 1993$.

[11] X. Li and J. Hwu, "A frequency hopping spread spectrum transmission scheme for uncoordinated cognitive radios," in Acoustics, Speech and Signal Processing, 2009, pp. 2345-2348.

[12] Digi. (17 April 2012). International. Available: http://www.digi.com/products/wireless-modems-peripherals/

[13] D. Digi International. (2007, 10 November 2011). Product Manual for 9XCite-PKG-R RM RS-232/485 RF Modem. Available: http://ftp1.digi.com/support/documentation/productmanual_xcite_pk gr_rs232rs485rfmodem.pdf

[14] J. G. M. Muhammad Ali Mazidi, "The 8051 Microcontroller Programming and Embedded Systems," United Kingdom, 1999.

[15] B. Mehta, "Interface LCD with ease," presented at the EDN, 2000.

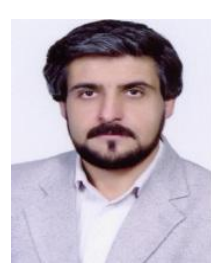

Fathollah Amouzegar received the degree in industrial engineering and the Master degree in industrial computing from the University Kebangssan Malaysia (UKM), Bangi, Kuala Lumpur, in 2012. He is studying in the field of Radio Frequency Identification (RFID).

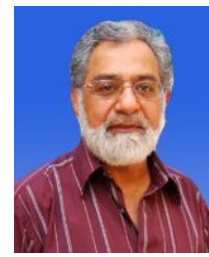

Ahmed Patel received his MSc. and PhD. degrees in Computer Science from Trinity College Dublin (TCD). $\mathrm{He}$ is Professor of Computer Science at Universiti Kebangsaan Malaysia \& Visiting Professor at Kingston University, UK. His research covers networking, security, digital forensics, autonomic computing and cloud computing. He has published 220 papers and co-authored several books. 